\title{
GC-MS Assisted Phytoactive Chemical Compounds Identification and Profiling with Mineral Constituents from Biologically Active Extracts of Aerva javanica (Burm. f) Juss. ex Schult.
}

\author{
Kandhan KARTHISHWARAN ${ }^{1,2}$, Subban KAMALRAJ ${ }^{3}$, \\ Chelliah JAYABASKARAN ${ }^{3}$, Shyam S. KURUP ${ }^{1}$, Sabitha SAKKIR ${ }^{4}$, \\ Abdul J. CHERUTH ${ }^{1 *}$
}

\author{
${ }^{1}$ United Arab Emirates University, College of Food and Agriculture, Department of Aridland Agriculture, Al Ain 15551, \\ UnitedArabEmirates; kandhan_k@uaeu.ac.ae; skurup@uaeu.ac.ae; abdul.jaleel@uaeu.ac.ae (*correspondingauthor) \\ ${ }^{2}$ United Arab Emirates University, College of Medicine and Health Sciences, Department of Pathology, Al Ain 15551, United Arab Emirates \\ ${ }^{3}$ Indian Institute of Science, Division of Biological Science, Department of Biochemistry, \\ Bangalore, Karnataka 560 012, India; cjb@biochem.iisc.ernet.in
}

${ }^{4}$ Wildlife Assessment and Conservation, Terrestrial and Marine Biodiversity Sector, Environment Agency-Abu Dhabi, P.O. Box 45553,

AbuDhabi,UnitedArabEmirates; ssakkir@ead.ae

\begin{abstract}
Aerva javanica (Burm. f) Juss. ex Schult. (Family: Amaranthaceae) family is one of the traditional medicinal plant growing in the United Arab Emirates. Apart from studies related to some medicinal properties, phytochemical, GC MS compound characterization and biological activities still to be investigated. An experiment was conducted to determine the possible bioactive components with their chemical structures and elucidation of phytochemicals from the aerial parts of the plant. The macro and micro-mineral constituents and antioxidant activities were also evaluated. Aerial parts of $A$. javanica were extracted sequentially with hexane, chloroform, ethyl acetate, acetone, methanol by cold percolation method. Free radical scavenging and antioxidant properties of methanolic extract were evaluated by using in vitro antioxidant assays such as hydroxyl radical scavenging activity, 1,1-diphenyl-2-picrylhydrazyl (DPPH) radicals, superoxide radical scavenging activity and ABTS radical scavenging activity. Primary phytochemical and micro-macro element was tested using standard protocol. The chemical characterization was done with the help of Gas Chromatography-Mass Spectrometry (GC-MS), and the mass spectra of the total compounds in the extract were matched with the National Institute of Standard and Technology (NIST) library. Mineral constituents were identified and estimated by ICP-OES. Ninety-nine metabolites were obtained by GC-MS anslysis; indole was found to be major components followed by 2-Chlorallyl diethyldithiocarbamate (CDEC), Carbaril, Bis(2ethylhexyl) phthalate, Quinoline, 4H-Cyclopenta[def] phenanthrene,2-[Bis(2-chloroethylamino)]-tetrahydro-2H-1,3,2oxazaphosphorine-2-oxide, Phenobarbital, 1H-Indole, 2-methyl-, 2,3,7,8-Tetrachlorodibenzo-p-dioxin Disulfide, diphenyl. The presence of various bioactive compounds in the extract validates the traditional medicinal uses of this plant.
\end{abstract}

Keywords: Aerva javanica; antioxidant; GC-MS; piperidine; Phenobarbital; phytochemical

\section{Introduction}

Day by day new drugs are being developed from different plant species. Highly significant anticancer drugs and different antimicrobial agents are the need of the day. The potential of herbal agents should be analyzed for their candidature in treatment of various ailments (Malongane $e t$ al., 2017). With the all the benefits of modern synthetic medicine, still people prefer plant based natural medicines due to the side effects of synthetic medicines (de Oliveira $e t$ al., 2017). Plants deliver dynamic molecule, develop at different degrees in many nations of the world, and are the premise of nearby traditional herbal medicine and drug in their particular societies (Teschke and Zhang, 2015). The synthetic additives and antioxidants are widely used in the current industries and health systems. In modern times, there are many food antioxidant substances and are 
518

harmless if used in minor quantities but in large quantities they have risks to human health like skin rashes and itching, and many other ailments (Lidon and Silva, 2016).

In the search for new compounds, and also for quality control, the suitable methods of screening are very important (Keskes et al., 2017). The selection of accurate medicinal plants, the extract screening within vitro methods for estimation of its biological activities will lead to the discovery of potentially useful compounds (Mathekaga and Meyer, 1998). In traditional medical systems, almost all the plants used may contain potential substances which are responsible for their biological activities and can be utilized for various diseases (Duraipandiyan et al., 2006). History reveals that plants are sources of successful drugs, and will continuously be important for screening of new lead compounds (Atanasov et al., 2015).

Aerva javanica (Burm. f) Juss. ex Schult. is one among the important plants in Amaranthaceae family. This plant is widely seen many countries (Judd et al., 2008). Many phytochemical compounds have been isolated from this plant (Emam, 1999). A. javanica has been widely used for many diseases in traditional and modern medical systems (Deshmukh et al., 2008). There are various reports stating the medicinal properties of $A$. javanica like antioxidant, antiviral, antiplasmodial, antibacterial (Baltina et al., 2003; Al-Fatimi et al., 2007; El-Hadi et al., 2010; Singh et al., 2010; Srinivas and Reddy, 2012). There are traditional uses for this plant (Samejo et al., 2012). The leaves of plant are used for fodder to goats and whole plant is used as a fuel. The whole $A$. javanica plant is used for the purpose of chest pain, ascaris and diarrhea with blood (Teklehaymanot and Giday, 2010).

In this study, there was analyzed the metabolic profile of $A$. javanica using gas chromatography-mass spectrometry (GC-MS) with their chemical structures and elucidation of phytochemicals from the aerial parts of the plant. The macro and micro-mineral constituents and antioxidant activities were also evaluated.

\section{Materials and Methods}

\section{Plantmaterial}

Samples of Aerva javanica (Burm. f) Juss. ex Schult. were collected from the Emirate of Abu Dhabi (UAE). The plant material collects in all collection site was authorized by the Department of Biology (College of Science, UAE University). The field studies did not involve endangered or protected species.

\section{Preparation of plant extracts}

The coarsely powdered the aerial parts of $A$. javanica were extracted sequentially with hexane, chloroform, ethyl acetate, acetone, methanol by cold percolation method. All the organic extract extracts were filtered using rotary evaporator. The residues were dissolved in Dimethyl Sulfoxide (DMSO) and these crude extracts were subjected to in-vitro assays. Different organic extract of samples for metabolomics analysis.

\section{Antioxidant activity of extracts}

$\mathrm{ABTS}^{\bullet+}$ radical (Wolfenden and Willson 1982) $\mathrm{DPPH}^{\bullet}$ assay (Brand-Williams et al., 1995), Superoxide anion scavenging activity (Nishimiki et al., 1972) and hydroxyl radical scavenging activity (Halliwell, 1982) were determined from the extracts.

\section{Phytochemical screening test for the extracts}

The dried extracts were subjected to the preliminary phytochemical screening by using Harbourne (Harborne, 1998) methods to test for alkaloids, flavonoids, tannins, terpenoids, steroids, saponins, protein, cardiac glycosides and anthraquinones.

\section{Microelements and macroelements}

Samples were prepared as explained previously (Ameri et al., 2014). The percentages of each element in this sample was determined by plotting the absorbance of each element versus the element concentration by using standard $A R$ grade solutions of the elements, for example $\mathrm{K}, \mathrm{Mg}, \mathrm{Ca}, \mathrm{Na}$, $\mathrm{Fe}, \mathrm{Mn}, \mathrm{Zn}, \mathrm{P}$ and S. The elemental analysis was done for aerial parts of the plant species (ICP-OES - Agilent Technologies, 710).

\section{Sample preparation for GCMS}

Different solvents (methanol, acetone, ethyl acetate, chloroform and Hexane) separately received and stored at $20^{\circ} \mathrm{C}$ for further use. For GCMS, each extract was prepared separately by dissolving $1 \mathrm{mg}$ of each extract dissolved in $1 \mathrm{ml}$ of fresh HPLC grade solvent and, vortexes for $2 \mathrm{~min}$ and the extract was filtered by $0.2 \mu l$ filter. The $25 \mathrm{ng} / \mu \mathrm{l}$ concentration each extract made with HPLC grade solvents was added $1 \mathrm{ml}$ of extract in respective GC tube for analysis. Methanol, acetone and chloroform samples were completely dried using Nitrogen gas. Sample was derivatised using $30 \mu \mathrm{l}$ pyridine and 60ul of BSTFA: TMCS (99:1) and incubated at $60{ }^{\circ} \mathrm{C}$ for $60 \mathrm{~min}$. Derivatised samples were subjected $1 \mu$ Lin to GC-MS (Lisec et al., 2006).

\section{GC-MS analysis}

GC-MS measurements were performed with a Shimadzu instrument equipped with GC: Aligent 7890 A, MS: MS detector 5975C, Ionization for MS: Electron Impact Ionization, Mass Analyzer: Single Quadrupole, Software: Data Analysis, AMDS, Library: NIST 2011, column: DP $5 \mathrm{~ms}$, Dimensions: $30 \mathrm{~m} \mathrm{~L} \mathrm{X} 0.25 \mathrm{~mm}$ ID $\times$ $0.25 \mu \mathrm{m}$ film thickness, initial temperature is $240{ }^{\circ} \mathrm{C} 2 \mathrm{~min}$ hold time, ram temperature is $50{ }^{\circ} \mathrm{C}$ to $280{ }^{\circ} \mathrm{C} 5 \mathrm{~min}$ is the hold time, total time is $40 \mathrm{~min}$, carrier gas is helium, flow $(13 \mathrm{ml} / \mathrm{min})$ is 1.0 , split flow: $1.5 \mathrm{ml} / \mathrm{min}$, injection volume: $1 \mu \mathrm{l}$, Scan mass range: $30 \mathrm{~m} / \mathrm{z}-600 \mathrm{~m} / \mathrm{z}$ and polarity $+\mathrm{ve}$. GC-MS performed based on the database having more than many patterns. The spectrum of the unknown compound was compared with the spectrum of the known compounds in library.

\section{Data analysis}

Raw GC-MS data were analysed as explained by Jin et al. (2016) and following the method of Jumtee et al. (2009). 


\section{Results and Discussion}

\section{Antioxidant assays of methanolic extract}

The extracts showed significant antioxidant potential when compared to standard gallic acid (Table 1). In ABTS+ scavenging activity the values are varied significantly $(\mathrm{P}<0.05)$ and ranged from 10 to $160 \mu \mathrm{g}$ GAE/g extract. The hydrogen-donating ability is revealed by this assay. The antioxidant activity was almost comparable directly to the standard gallic acid (IC50 value $7.94 \mu \mathrm{g} / \mathrm{mL}$ ) (Table 1). Gallic acid was used as reference standard in DPPH method. In comparison with gallic acid, the methanolic extracts of $A$. javanica showed high scavenging activity in vitro (Table 1 ). javanica was 39.1-76.4\% and that of the standard gallica was $37.2-58.3 \%$. The methanolic extract of $A$. javanica exhibited concentration-dependent radical scavenging activity, that is, percentage inhibition increased with sample concentration (Table 1).

\section{Phytochemical screening of methanolic extract}

Phytochemical screening for the methanolic extracts of $A$. javanica reveals the active phytochemical constituents of the plant understudy in which flavonoids, tannins, alkaloids, steriods, protein, terpenoid, phenols, cardiac glycosidel were present. However, the extract tested showed the absence of saponin and anthraquinones (Table 2).

Table 1. In vitro free radical scavenging activities of methanolic extract from Aerva javanica

\begin{tabular}{|c|c|c|c|c|c|c|c|c|}
\hline \multirow{3}{*}{$\begin{array}{l}\text { Concentration } \\
\qquad(\mu \mathrm{g} / \mathrm{ml})\end{array}$} & \multicolumn{8}{|c|}{ Inhibitory activity (\%) } \\
\hline & \multicolumn{2}{|c|}{$\begin{array}{c}\text { Hydroxyl radical scavenging } \\
\text { activity }\end{array}$} & \multicolumn{2}{|c|}{$\begin{array}{l}\text { Superoxide radical scavenging } \\
\text { activity }\end{array}$} & \multicolumn{2}{|c|}{$\begin{array}{c}\text { DPPH radical scavenging } \\
\text { activity }\end{array}$} & \multicolumn{2}{|c|}{$\begin{array}{c}\text { ABTS radical scavenging } \\
\text { activity }\end{array}$} \\
\hline & $\begin{array}{c}\text { Gallic acid } \\
(G A E)\end{array}$ & A. javanica & $\begin{array}{c}\text { Gallic acid } \\
\text { (GAE) }\end{array}$ & A. javanica & $\begin{array}{c}\text { Gallic acid } \\
(G A E)\end{array}$ & A. javanica & $\begin{array}{c}\text { Gallic acid } \\
(G A E)\end{array}$ & A.javanica \\
\hline 10 & $41.0 \pm 2.8$ & $27.3 \pm 1.4$ & $37.2 \pm 1.6$ & $39.1 \pm 1.2$ & $38.2 \pm 2.8$ & $46.2 \pm 1.1$ & $36.5 \pm 2.8$ & $52.3 \pm 2.3$ \\
\hline 20 & $42.3 \pm 1.3$ & $36.2 \pm 2.1$ & $50.4 \pm 1.8$ & $44.6 \pm 1.7$ & $43.1 \pm 1.4$ & $57.5 \pm 2.6$ & $40.4 \pm 1.9$ & $55.2 \pm 2.0$ \\
\hline 40 & $48.9 \pm 1.5$ & $46.1 \pm 1.6$ & $53.1 \pm 2.9$ & $59.4 \pm 2.5$ & $56.2 \pm 2.9$ & $65.8 \pm 3.3$ & $43.9 \pm 3.4$ & $66.6 \pm 1.9$ \\
\hline 80 & $51.0 \pm 2.4$ & $56.4 \pm 2.9$ & $55.3 \pm 1.2$ & $65.2 \pm 3.6$ & $62.4 \pm 1.6$ & $74.1 \pm 2.8$ & $48.0 \pm 2.5$ & $68.2 \pm 1.2$ \\
\hline 160 & $54.0 \pm 3.2$ & $62.1 \pm 2.4$ & $58.3 \pm 3.2$ & $76.4 \pm 3.3$ & $67.5 \pm 2.1$ & $83.1 \pm 1.6$ & $60.3 \pm 3.2$ & $78.5 \pm 2.5$ \\
\hline IC 50 & $36.8 \pm 1.1$ & $35.5 \pm 1.4$ & $33.96 \pm 1.1$ & $28.96 \pm 0.5$ & $31.25 \pm 0.8$ & $25.69 \pm 1.4$ & $7.94 \pm 0.1$ & $5.35 \pm 0.3$ \\
\hline
\end{tabular}

Data represented as means $\pm S D(n=5)$.

Both A. javanica and GAE showed significant inhibitory activity in a concentration-dependent manner to inhibit hydroxyl-radical-mediated deoxyribose damage was assessed at a concentration of $10-160 \mu \mathrm{g} / \mathrm{mL}$. In the present investigation, the hydroxyl radical scavenging activity observed was in the range of $27.3-62.1 \%$ at the concentration of $160 \mu \mathrm{g} / \mathrm{ml}$. While scavenging hydroxyl radical, the ability of methanol extract (62.1\%) was found to be higher than reference standard gallic acid (54.0\%) (Table 1). Superoxide radical reduced NBT to a blue-colored formation that was measured at $560 \mathrm{~nm}$. At 10-160 $\mu \mathrm{g}$, the superoxide scavenging activity of methanolic extract of $A$.

Table 2. Preliminary phytochemical constituents of Aerva javanica plants

\begin{tabular}{cc}
\hline Constituent & Concentration \\
\hline Flavonoid & ++ \\
Tannin & + \\
Alkaloid & + \\
Steroids & + \\
Protein & + \\
Terpenoids & + \\
Saponin & + \\
Phenols & ++ \\
Cardiac glycoside & + \\
\hline $\begin{array}{l}\text { Legend: } \\
+=\text { Low concentration; } \\
++=\text { Moderate concentration; } \\
+++ \text { High concentration; } \\
-=\text { Absent. }\end{array}$ & \\
&
\end{tabular}

\section{Mineral compositions}

Nutritional composition of the aerial plant parts of $A$. javanica was presented in Table 3. $\mathrm{Ca}, \mathrm{Cr}, \mathrm{Cu} \mathrm{K}, \mathrm{Mg}, \mathrm{Mn}$, $\mathrm{Ni}, \mathrm{P}, \mathrm{S}$ and $\mathrm{Zn}$ were presented in appreciable quantities. Low concentrations of $\mathrm{Cd}, \mathrm{Co}, \mathrm{Fe}, \mathrm{N}$, and $\mathrm{Pb}$ were observed in the methanolic extract of $A$. javanica.

Table 3. Mineral composition of Aerva javanica plants (mg/kg (ppm))

\begin{tabular}{cc}
\hline $\begin{array}{c}\text { Mineral } \\
\text { composition }\end{array}$ & $\begin{array}{c}\text { Content } \\
(\mathrm{mg} / \mathrm{kg}(\mathrm{ppm}))\end{array}$ \\
\hline $\mathrm{Ca}$ & 17.34 \\
$\mathrm{Cd}$ & $<0.001$ \\
$\mathrm{Co}$ & 0.58 \\
$\mathrm{Cr}$ & 5.2 \\
$\mathrm{Cu}$ & 3.29 \\
$\mathrm{Fe}$ & 0.38 \\
$\mathrm{~K}$ & 11.58 \\
$\mathrm{Mg}$ & 10.7 \\
$\mathrm{Mn}$ & 23.3 \\
$\mathrm{Ni}$ & 6.57 \\
$\mathrm{Na}$ & 0.83 \\
$\mathrm{~Pb}$ & $<0.011$ \\
$\mathrm{P}$ & 1.64 \\
$\mathrm{~S}$ & 2.6 \\
$\mathrm{Zn}$ & 15.6 \\
\hline
\end{tabular}


Table 4. Biologically active chemical compounds of hexane extract of the aerial parts of $A$. javanica

\begin{tabular}{|c|c|c|c|c|c|c|}
\hline No & CAS & $\mathrm{RT}(\min )$ & $\begin{array}{l}\text { Name of the } \\
\text { compound }\end{array}$ & $\begin{array}{l}\text { Molecular } \\
\text { formula }\end{array}$ & $\begin{array}{c}\text { Molecular } \\
\text { weight }\end{array}$ & $\begin{array}{c}\text { Peak area } \\
(\%)\end{array}$ \\
\hline 1 & $591-78-6$ & 4.3073 & 2-Hexanone & $\mathrm{CH}_{3}\left(\mathrm{CH}_{2}\right)_{3} \mathrm{COCH}_{3}$ & 100.16 & 0.39 \\
\hline 2 & $1678-91-7$ & 5.1103 & $\begin{array}{c}\text { Cyclohexane, ethyl- - incomplete } \\
\text { name }\end{array}$ & & 112.21 & 1.00 \\
\hline 3 & $110-89-4$ & 5.4264 & Piperidine & $\mathrm{C}_{5} \mathrm{H}_{11} \mathrm{~N}$ & 85.15 & 3.60 \\
\hline 4 & $95-47-6$ & 6.1992 & o-Xylene & $\mathrm{C}_{6} \mathrm{H}_{4}\left(\mathrm{CH}_{3}\right)_{2}$ & 106.17 & 2.50 \\
\hline 5 & $98-82-8$ & 6.8798 & $\begin{array}{l}\text { Benzene, (1-methylethyl)- - } \\
\text { incomplete name }\end{array}$ & $\mathrm{C}_{6} \mathrm{H}_{5} \mathrm{CH}\left(\mathrm{CH}_{3}\right)_{2}$ & 120.19 & 8.50 \\
\hline 6 & $103-65-1$ & 7.5958 & Benzene, propyl- - incomplete name & $\mathrm{C}_{6} \mathrm{H}_{5} \mathrm{CH}_{2} \mathrm{CH}_{2} \mathrm{CH}_{3}$ & 120.19 & 4.70 \\
\hline 7 & $611-14-3$ & 7.7825 & $\begin{array}{l}\text { Benzene, 1-ethyl-2-methyl- - } \\
\text { incomplete name }\end{array}$ & $\mathrm{C}_{9} \mathrm{H}_{12}$ & 120.19 & 3.30 \\
\hline 8 & $108-67-8$ & 8.6278 & $\begin{array}{l}\text { Benzene, 1,3,5-trimethyl-- } \\
\text { incomplete name }\end{array}$ & $\mathrm{C}_{6} \mathrm{H}_{3}\left(\mathrm{CH}_{3}\right)_{3}$ & 120.19 & 5.70 \\
\hline 9 & $629-50-5$ & 8.7567 & Tridecane & $\mathrm{CH}_{3}\left(\mathrm{CH}_{2}\right)_{11} \mathrm{CH}_{3}$ & 184.36 & 5.70 \\
\hline 10 & $124-18-5$ & 8.7635 & Decane & $\mathrm{CH}_{3}\left(\mathrm{CH}_{2}\right)_{8} \mathrm{CH}_{3}$ & 142.28 & 0.01 \\
\hline
\end{tabular}

\section{GC-MS Analysis of different solvents extract}

From five batches of approximately $1 \mathrm{~kg}$ of air dried powdered leaves, mean percentage yields of $4.78 \%$ of $n$ hexane extract, $5.12 \%$ of chloroform ethyl acetate extract, $3.52 \%$ of Ethyl acetate, $2.74 \%$ of acetone extract and $8.47 \%$ of methanol extract were obtained. Methanol extract gave the highest percentage yield. Most of the constituents were polar in nature.

There are many unknown compounds in the polar extracts from the plant material of $A$. javanica. Comprehensive coverage showed similar discrimination as when using the 155 known and putative metabolites and solely the 99 identified features.

It is for the first time that the composition of the five extracts of the aerial parts of $A$. javanica has been investigated through GC / MS analysis. The mass spectra were compared with those of the known compounds stored in the National Institute of Standards and Technology (NIST) research library. The bioactive compounds present in n-hexane, chloroform, ethyl acetate, acetone and methanol extracts obtained from $A$. javanica aerial parts are shown in Tables 1-5. The prevailing compounds were 2Chlorallyl diethyldithiocarbamate (CDEC), Carbaril, Bis(2-ethylhexyl) phthalate, Quinoline, $4 \mathrm{H}$ Cyclopenta[def]phenanthrene,

chloroethylamino)]-tetrahydro-2H-1,3,2-

oxazaphosphorine-2-oxide, Phenobarbital, 1H-Indole, 2methyl-, 2,3,7,8-Tetrachlorodibenzo-p-dioxin, Disulfide, diphenyl.

As whole Hexane extract of the aerial parts of $A$. javanica was found to be rich in benzene metabolites. On comparison of the mass spectra of the constituents with the NIST library, the ten phytocompounds were characterized and identified (Table 4). The results revealed that Piperidine was found as the one major component is the hexane extract and the nine minor components are Tridecane, o-Xylene, 2-Hexanone and other benzene derivatives were also present.

The chloroform extract of $A$. javanica pertaining to the GC-MS analysis were given in Table 5. Thirty-eight compounds were detected in the chloroform extract of the aerial parts of $A$. javanica and Piperidine, Carbaril, Bis(2ethylhexyl) phthalate, Quinoline were found to be the major compounds followed by Dimethyl phthalate, Propanedinitrile, 5-Fluorouracil, 1,3-Dioxolane, Eicosane and various benzene and propenoic acid were also identified.

The ethyl acetate extracts showed twenty chemical compounds in the GC MS analysis (Table 6) which were detected according to their retention time on fused silica capillary column. These compounds mainly comprised of oXylene, Dimethyl sulfon, Undecane, Pentadecane was identified as a major chemical constituent followed by Propanoic acid, Ethylbenzene Tridecane, Dibutyl phthalate etc.

The most polar methanol extract of the aerial parts of $A$. javanica led to the identification of nineteen chemical compounds by GC MS analysis (Table 7). 1H-Indole, 2methyl-, CDEC, 2,3,7,8-Tetrachlorodibenzo-p-dioxin, Disulfide, diphenyl and Benzothiazole, 2-methyl- were identified as the main chemical constituents followed fourteen minor components are Naphthalene, 1,2dimethyl, Thiotepa, Sulfur tetrafluoride2Imidazolidinethione and other benzene derivatives were also identified.

The acetone ether crude extract of the aerial parts of $A$. javanica were analysed by GC-MS which leads to the identification of 17 organic compounds. The chemical compounds were identified according to their retention time on a fused silica capillary column. The list of the compounds with their retention time, molecular formula, molecular weight, area and area (\%) were presented in Table 8. The identified major compounds are $4 \mathrm{H}$ Cyclopenta[def]phenanthrene, CDEC, 2-[Bis(2chloroethylamino)]-tetrahydro-2H-1,3,2-

oxazaphosphorine-2-oxide, Phenobarbital along with other minor constituents were also present.

The antioxidant properties of this plant extracts can be attributed to the presence of different phytochemicals. In this context, the presence of phytochemicals such as alkaloids, flavonoids, tannins, steroids, terpenoids, reducing sugar, saponins and glycosides are to be mentioned, as they 
have a prominent role in plant antioxidant responses (Robert and Lin, 1994). The presence of flavonoid itself function acts as scavenger of free radicals by rapid donation of a hydrogen atom affording a phenoxy radical intermediate that is relatively stable (Bors et al., 1990). Similar antioxidant actions and phytochemicals were reported previously from $A$. lanata (Yamunadevi et al., 2001; Kumar et al., 2013). Crude extracts of different parts of $A$. javanica made with hexane, chloroform and methanol showed the presence of phytochemicals (Srinivas and Reddy, 2012).
Different extracts were obtained from the aerial parts of A. javanica through selective sequential extraction with solvents of increasing polarity, namely, n-hexane, chloroform, ethyl acetate, acetone and methanol. GC-MS analysis of the n-hexane, ethyl acetate and methanol extracts revealed the presence of various bioactive compounds.

The major compound of Piperidine is present both in Hexane and chloroform extracts but in different quantities. In addition, Phenobarbital is present both acetone and the polar methanol extract. Piperidine are significant as they are

Table 5. Biologically active chemical compounds of chloroform extract of the aerial parts of $A$. javanica

\begin{tabular}{|c|c|c|c|c|c|c|}
\hline No & CAS & $\mathrm{RT}(\mathrm{min})$ & $\begin{array}{l}\text { Name of the } \\
\text { compound }\end{array}$ & $\begin{array}{l}\text { Molecular } \\
\text { formula }\end{array}$ & $\begin{array}{c}\text { Molecular } \\
\text { weigh }\end{array}$ & $\begin{array}{c}\text { Peak area } \\
(\%)\end{array}$ \\
\hline 1. & $110-86-1$ & 4.0984 & Pyridine & $\mathrm{C}_{5} \mathrm{H}_{5} \mathrm{~N}$ & 79.10 & 0.01 \\
\hline 2. & $106-46-7$ & 4.2902 & Benzene, 1,4-dichloro- - incomplete name & $\mathrm{C}_{6} \mathrm{H}_{4} \mathrm{Cl}_{2}$ & 147.002 & 9.40 \\
\hline 3. & $107-20-0$ & 5.3032 & Acetaldehyde, chloro- - incomplete name & $\mathrm{C}_{2} \mathrm{H}_{3} \mathrm{ClO}$ & 78.498 & 0.00 \\
\hline 4. & $131-11-3$ & 5.3148 & Dimethyl phthalate & $\mathrm{C}_{6} \mathrm{H}_{4}-1,2-\left(\mathrm{CO}_{2} \mathrm{CH}\right)$ & 194.18 & 0.27 \\
\hline 5. & $556-64-9$ & 5.3148 & Thiocyanic acid, methyl ester & $\mathrm{C}_{2} \mathrm{H}_{3} \mathrm{NS}$ & 73.12 & 0.01 \\
\hline 6. & $109-77-3$ & 8.1947 & Propanedinitrile & $\mathrm{CH}_{2}(\mathrm{CN})_{2}$ & 66.06 & 16.00 \\
\hline 7. & $83-34-1$ & 8.557 & 1H-Indole, 3-methyl- - incomplete name & $\mathrm{C}_{9} \mathrm{H}_{9} \mathrm{~N}$ & 131.17 & 0.07 \\
\hline 8. & $51-21-8$ & 8.5577 & 5-Fluorouracil & $\mathrm{C}_{4} \mathrm{H}_{3} \mathrm{FN}_{2} \mathrm{O}_{2}$ & 130.08 & 0.09 \\
\hline 9. & $56-04-2$ & 8.5832 & Myethlthiouracil & $\mathrm{C}_{5} \mathrm{H}_{6} \mathrm{~N}_{2} \mathrm{OS}$ & 142.18 & 2.60 \\
\hline 10. & $2097-19-0$ & 8.6349 & $\begin{array}{l}\text { 2,8,9-Trioxa-5-aza-1- } \\
\text { silabicyclo[3.3.3] undecane, 1-phenyl-- } \\
\text { incomplete name }\end{array}$ & $\mathrm{C}_{12} \mathrm{H}_{17} \mathrm{NO}_{3} \mathrm{Si}$ & 251.35 & 0.01 \\
\hline 11. & $4170-30-3$ & 8.6454 & 2-Butenal & $\mathrm{C}_{4} \mathrm{H}_{6} \mathrm{O}$ & 70.09 & 0.11 \\
\hline 12. & $61-82-5$ & 8.8386 & 3-Amino-s-triazole & $\mathrm{C}_{2} \mathrm{H}_{4} \mathrm{~N}_{4}$ & 84.08 & 11.00 \\
\hline 13. & $2437-56-1$ & 8.8716 & 1-Tridecene & $\mathrm{CH}_{3}\left(\mathrm{CH}_{2}\right)_{10} \mathrm{CH}=\mathrm{CH}_{2}$ & 182.35 & 0.68 \\
\hline 14. & $104-76-7$ & 8.8754 & 1-Hexanol, 2-ethyl- - incomplete name & $\begin{array}{c}\mathrm{CH}_{3}\left(\mathrm{CH}_{2}\right)_{3} \mathrm{CH}\left(\mathrm{C}_{2} \mathrm{H}_{5}\right) \mathrm{CH}_{2} \mathrm{O} \\
\mathrm{H}\end{array}$ & 130.23 & 0.62 \\
\hline 15. & $141-79-7$ & 8.9026 & $\begin{array}{l}\text { 3-Penten-2-one, 4-methyl- - incomplete } \\
\text { name }\end{array}$ & $\mathrm{C}_{6} \mathrm{H}_{10} \mathrm{O}$ & 98.15 & 4.80 \\
\hline 16. & $98-00-0$ & 9.1111 & 2-Furanmethanol & $\mathrm{C}_{5} \mathrm{H}_{6} \mathrm{O}_{2}$ & 98.09 & 6.20 \\
\hline 17. & $203-64-5$ & 9.1431 & 4H-Cyclopenta[def]phenanthrene & $\mathrm{C}_{15} \mathrm{H}_{10}$ & 190.24 & 5.50 \\
\hline 18. & $123-38-6$ & 9.1699 & Propanal & $\mathrm{CH}_{3} \mathrm{CH}_{2} \mathrm{CHO}$ & 58.08 & 3.10 \\
\hline 19. & $767-59-9$ & 9.2468 & 1H-Indene, 1-methyl- & $\mathrm{C}_{10} \mathrm{H}_{10}$ & 130.19 & 6.90 \\
\hline 20. & $2682-20-4$ & 9.4785 & $\begin{array}{c}\text { 3(2H)-Isothiazolone, 2-methyl- - incomplete } \\
\text { name }\end{array}$ & $\mathrm{C}_{4} \mathrm{H}_{5} \mathrm{NOS}$ & 115.15 & 0.04 \\
\hline 21. & $624-83-9$ & 9.7424 & Methane, isocyanato- & $\mathrm{C}_{2} \mathrm{H}_{5} \mathrm{NO}$ & 57.05 & 2.60 \\
\hline 22. & $78-93-3$ & 9.9186 & 2-Butanone & $\mathrm{C}_{2} \mathrm{H}_{5} \mathrm{COCH}_{3}$ & 72.11 & 0.04 \\
\hline 23. & $621-82-9$ & 9.973 & $\begin{array}{l}\text { 2-Propenoic acid, 3-phenyl- - incomplete } \\
\text { name }\end{array}$ & $\mathrm{C}_{9} \mathrm{H}_{8} \mathrm{O}_{2}$ & 148.16 & 0.66 \\
\hline 24. & $121-44-8$ & 10.0356 & Triethylamnie & $\left(\mathrm{C}_{2} \mathrm{H}_{5}\right)_{3} \mathrm{~N}$ & 101.19 & 7.20 \\
\hline 25. & $259-79-0$ & 12.7068 & Biphenylene & $\mathrm{C}_{12} \mathrm{H}_{8}$ & 152.19 & 1.80 \\
\hline 26. & $606-20-2$ & 13.1986 & $\begin{array}{c}\text { Benzene, 2-methyl-1,3-dinitro- - incomplete } \\
\text { name }\end{array}$ & $\mathrm{C}_{7} \mathrm{H}_{6} \mathrm{~N}_{2} \mathrm{O}_{4}$ & 182.13 & 1.70 \\
\hline 27. & $63-25-2$ & 13.7694 & Carbaril & $\mathrm{C}_{10} \mathrm{H}_{7} \mathrm{OCONHCH}{ }_{3}$ & 201.22 & 20.00 \\
\hline 28. & $135-19-3$ & 13.7694 & 2-Naphthalenol & $\mathrm{C}_{10} \mathrm{H}_{7} \mathrm{OH}$ & 144.17 & 20.00 \\
\hline 29. & 646-06-0 & 14.9272 & 1,3-Dioxolane & $\mathrm{C}_{3} \mathrm{H}_{6} \mathrm{O}_{2}$ & 74.08 & 0.64 \\
\hline 30. & $883-20-5$ & 15.5789 & Phenanthrene, 9-methyl- - incomplete name & $\mathrm{C}_{15} \mathrm{H}_{12}$ & 192.26 & 10.00 \\
\hline 31. & $91-08-7$ & 16.1419 & $\begin{array}{l}\text { Benzene, 1,3-diisocyanato-2-methyl- - } \\
\text { incomplete name }\end{array}$ & $\mathrm{C}_{9} \mathrm{H}_{6} \mathrm{~N}_{2} \mathrm{O}_{2}$ & 174.16 & 25.00 \\
\hline 32. & $1910-42-5$ & 20.7712 & Paraquat dichloride & $\mathrm{C}_{12} \mathrm{H}_{14} \mathrm{Cl}_{2} \mathrm{~N}_{2} \cdot \mathrm{xH}_{2} \mathrm{O}$ & 257.16 & 7.70 \\
\hline 33. & $91-20-3$ & 20.7712 & Naphthalene & $\mathrm{C}_{10} \mathrm{H}_{8}$ & 128.17 & 36.00 \\
\hline 34. & $1490-04-6$ & 29.6864 & Menthol & $\mathrm{C}_{10} \mathrm{D}_{4} \mathrm{H}_{16} \mathrm{O}$ & 160.29 & 33.00 \\
\hline 35. & $629-59-4$ & 35.1042 & Tetradecane & $\mathrm{CH}_{3}\left(\mathrm{CH}_{2}\right)_{12} \mathrm{CH}_{3}$ & 198.39 & 20.00 \\
\hline 36. & $91-59-8$ & 36.0457 & 2-Naphthalenamine & $\mathrm{C}_{10} \mathrm{H}_{7} \mathrm{NH}_{2}$ & 143.19 & 9.50 \\
\hline 37. & $117-81-7$ & 41.8833 & Bis(2-ethylhexyl) phthalate & $\mathrm{C}_{24} \mathrm{H}_{38} \mathrm{O}_{4}$ & 390.56 & 1.30 \\
\hline 38. & $91-22-5$ & 42.2789 & Quinoline & $\mathrm{C}_{9} \mathrm{H}_{7} \mathrm{~N}$ & 129.16 & 12.00 \\
\hline
\end{tabular}


Table 6. Biologically active chemical compounds of ethyl acetate extract of the aerial parts of $A$. javanica

\begin{tabular}{|c|c|c|c|c|c|c|}
\hline No & CAS & $\begin{array}{l}\text { RT } \\
(\mathrm{min})\end{array}$ & $\begin{array}{l}\text { Name of the } \\
\text { compound }\end{array}$ & $\begin{array}{l}\text { Molecular } \\
\text { formula }\end{array}$ & $\begin{array}{c}\text { Molecular } \\
\text { weigh }\end{array}$ & $\begin{array}{c}\text { Peak area } \\
(\%)\end{array}$ \\
\hline 1. & $67-66-3$ & 4.2399 & Trichloromethane & $\mathrm{CHCl}_{3}$ & 119.38 & 0.00 \\
\hline 2. & $64-19-7$ & 4.2658 & Acetic acid & $\mathrm{CH}_{3} \mathrm{CO}_{2} \mathrm{H}$ & 60.05 & 0.00 \\
\hline 3. & $71-43-2$ & 4.2964 & Benzene & $\mathrm{C}_{6} \mathrm{H}_{6}$ & 78.11 & 0.61 \\
\hline 4. & $79-09-4$ & 4.3716 & Propanoic acid & $\mathrm{CH}_{3} \mathrm{CH}_{2} \mathrm{COOH}$ & 74.08 & 0.84 \\
\hline 5. & $108-24-7$ & 4.4855 & Acetic anhydride & $\left(\mathrm{CH}_{3} \mathrm{CO}\right)_{2} \mathrm{O}$ & 102.09 & 0.26 \\
\hline 6. & $103-81-1$ & 4.892 & Benzeneacetamide & $\mathrm{C}_{8} \mathrm{H}_{9} \mathrm{NO}$ & 135.16 & 0.13 \\
\hline 7. & $100-41-4$ & 6.1151 & Ethylbenzene & $\mathrm{C}_{6} \mathrm{H}_{5} \mathrm{C}_{2} \mathrm{H}_{5}$ & 106.17 & 3.00 \\
\hline 8. & $108-38-3$ & 6.1151 & $\begin{array}{l}\text { Benzene, 1,3-dimethyl- - incomplete } \\
\text { name }\end{array}$ & $\mathrm{C}_{6} \mathrm{H}_{4}\left(\mathrm{CH}_{3}\right)_{2}$ & 106.17 & 3.00 \\
\hline 9. & $95-47-6$ & 6.2631 & o-Xylene & $\mathrm{C}_{6} \mathrm{H}_{4}\left(\mathrm{CH}_{3}\right)_{2}$ & 106.17 & 4.10 \\
\hline 10. & $67-71-0$ & 6.972 & Dimethyl sulfone & $\left(\mathrm{CH}_{3}\right)_{2} \mathrm{SO}_{2}$ & 94.13 & 3.60 \\
\hline 11. & $1120-21-4$ & 11.7129 & Undecane & $\mathrm{CH}_{3}\left(\mathrm{CH}_{2}\right)_{9} \mathrm{CH}_{3}$ & 156.31 & 5.60 \\
\hline 12. & $112-42-5$ & 19.545 & 1-Undecanol & $\mathrm{CH}_{3}\left(\mathrm{CH}_{2}\right)_{10} \mathrm{OH}$ & 172.31 & 2.50 \\
\hline 13. & $128-37-0$ & 22.2767 & Butylated Hydroxytoluene & $\begin{array}{c}{\left[\left(\mathrm{CH}_{3}\right)_{3} \mathrm{C}\right]_{2} \mathrm{C}_{6} \mathrm{H}_{2}} \\
\left(\mathrm{CH}_{3}\right) \mathrm{OH}\end{array}$ & 220.35 & 0.35 \\
\hline 14. & $96-76-4$ & 22.34 & $\begin{array}{l}\text { Phenol, 2,4-bis(1,1-dimethylethyl)- - } \\
\text { incomplete name }\end{array}$ & $\mathrm{C}_{14} \mathrm{H}_{22} \mathrm{O}$ & 206.32 & 0.95 \\
\hline 15. & $36653-82-4$ & 28.8017 & 1-Hexadecanol & $\mathrm{CH}_{3}\left(\mathrm{CH}_{2}\right)_{15} \mathrm{OH}$ & 242.44 & 1.60 \\
\hline 16. & $84-74-2$ & 31.982 & Dibutyl phthalate & $\begin{array}{c}\mathrm{C}_{6} \mathrm{H}_{4-1,2-} \\
{\left[\mathrm{CO}_{2}\left(\mathrm{CH}_{2}\right)_{3} \mathrm{CH}_{3}\right]_{2}}\end{array}$ & 278.34 & 1.20 \\
\hline 17. & $57-10-3$ & 32.1447 & n-Hexadecanoic acid & $\mathrm{CH}_{3}\left(\mathrm{CH}_{2}\right)_{14} \mathrm{COOH}$ & 256.42 & 2.00 \\
\hline 18. & $544-76-3$ & 41.4625 & Hexadecane & $\mathrm{CH}_{3}\left(\mathrm{CH}_{2}\right)_{14} \mathrm{CH}_{3}$ & 226.44 & 2.30 \\
\hline 19. & $629-62-9$ & 41.471 & Pentadecane & $\mathrm{CH}_{3}\left(\mathrm{CH}_{2}\right)_{13} \mathrm{CH}_{3}$ & 212.41 & 2.60 \\
\hline 20. & $112-95-8$ & 44.4415 & Eicosane & $\mathrm{CH}_{3}\left(\mathrm{CH}_{2}\right)_{18} \mathrm{CH}_{3}$ & 282.55 & 1.40 \\
\hline
\end{tabular}

Table 7. Biologically active chemical compounds of methanol extract of the aerial parts of $A$. javanica

\begin{tabular}{|c|c|c|c|c|c|c|}
\hline No & CAS & $\mathrm{RT}(\mathrm{min})$ & $\begin{array}{l}\text { Name of the } \\
\text { compound }\end{array}$ & $\begin{array}{l}\text { Molecular } \\
\text { formula }\end{array}$ & $\begin{array}{c}\text { Molecular } \\
\text { weight }\end{array}$ & $\begin{array}{c}\text { Peak area } \\
(\%)\end{array}$ \\
\hline 1. & $101-55-3$ & 9.648 & $\begin{array}{l}\text { Benzene, 1-bromo-4-phenoxy- } \\
\text { incomplete name }\end{array}$ & $\mathrm{C}_{12} \mathrm{H}{ }_{9} \mathrm{BrO}$ & 249.1 & 14.00 \\
\hline 2. & $74-85-1$ & 9.9249 & Ethylene & $\mathrm{CH}_{2}=\mathrm{CH}_{2}$ & 28.05 & 0.42 \\
\hline 3. & $96-45-7$ & 10.0933 & 2-Imidazolidinethione & $\mathrm{C}_{3} \mathrm{H}_{6} \mathrm{~N}_{2} \mathrm{~S}$ & 102.16 & 0.90 \\
\hline 4. & $608-27-5$ & 12.8294 & $\begin{array}{l}\text { Benzenamine, 2,3-dichloro-- incomplete } \\
\text { name }\end{array}$ & $\mathrm{C}_{6} \mathrm{H}_{5} \mathrm{Cl}_{2} \mathrm{~N}$ & 162.02 & 11.00 \\
\hline 5. & $7783-60-0$ & 13.8345 & Sulfur tetrafluoride & $\mathrm{F}_{4} \mathrm{~S}$ & 108.06 & 12.00 \\
\hline 6. & $120-72-9$ & 14.8213 & Indole (antioxidant) & $\mathrm{C}_{8} \mathrm{H}_{7} \mathrm{~N}$ & 117.15 & 8.50 \\
\hline 7. & $1634-04-4$ & 14.9315 & $\begin{array}{l}\text { Propane, 2-methoxy-2-methyl- - } \\
\text { incomplete name }\end{array}$ & $\mathrm{C}_{5} \mathrm{H}_{12} \mathrm{O}$ & 88.14 & 0.57 \\
\hline 8. & $52-24-4$ & 15.5451 & Thiotepa & $\mathrm{C}_{6} \mathrm{H}_{12} \mathrm{~N}_{3} \mathrm{PS}$ & 189.22 & 0.97 \\
\hline 9. & $613-12-7$ & 15.5631 & Anthracene, 2-methyl- - incomplete name & $\mathrm{C}_{15} \mathrm{H}_{12}$ & 192.26 & 11.00 \\
\hline 10. & $95-83-0$ & 16.9513 & $\begin{array}{l}\text { 1,2-Benzenediamine, 4-chloro- - } \\
\text { incomplete name }\end{array}$ & $\mathrm{ClC}_{6} \mathrm{H}_{3}\left(\mathrm{NH}_{2}\right)_{2}$ & 142.59 & 6.00 \\
\hline 11. & $95-06-7$ & 17.7567 & CDEC & $\mathrm{C}_{8} \mathrm{H}_{14} \mathrm{ClNS}_{2}$ & 223.79 & 4.70 \\
\hline 12. & $573-98-8$ & 20.7874 & $\begin{array}{c}\text { Naphthalene, 1,2-dimethyl- - incomplete } \\
\text { name }\end{array}$ & $\mathrm{C}_{12} \mathrm{H}_{12}$ & 156.22 & 8.00 \\
\hline 13. & $1746-01-6$ & 29.6141 & 2,3,7,8-Tetrachlorodibenzo-p-dioxin & $\mathrm{C}_{12} \mathrm{H}_{4} \mathrm{Cl}$ & 321.97 & 0.95 \\
\hline 14. & $882-33-7$ & 31.6179 & Disulfide, diphenyl & $\mathrm{C}_{6} \mathrm{H}_{5} \mathrm{SSC}_{6} \mathrm{H}_{5}$ & 218.34 & 1.50 \\
\hline 15. & $79-92-5$ & 36.7619 & Camphene & $\mathrm{C}_{10} \mathrm{H}_{16}$ & 136.23 & 7.10 \\
\hline 16. & $7005-72-3$ & 37.1718 & $\begin{array}{l}\text { Benzene, 1-chloro-4-phenoxy- - } \\
\text { incomplete name }\end{array}$ & $\mathrm{C}_{12} \mathrm{H}_{9} \mathrm{ClO}$ & 204.65 & 9.60 \\
\hline 17. & $120-75-2$ & 46.0812 & Benzothiazole, 2-methyl- & $\mathrm{C}_{8} \mathrm{H}_{7} \mathrm{NS}$ & 149.21 & 8.80 \\
\hline 18. & $84-76-4$ & 46.3526 & $\begin{array}{l}\text { 1,2-Benzenedicarboxylic acid, dinonyl } \\
\text { ester }\end{array}$ & $\mathrm{C}_{26} \mathrm{H}_{42} \mathrm{O}_{4}$ & 418.61 & 7.10 \\
\hline 19. & $84-69-5$ & 46.353 & $\begin{array}{c}\text { 1,2-Benzenedicarboxylic acid, bis(2- } \\
\text { methylpropyl) ester }\end{array}$ & $\mathrm{C}_{16} \mathrm{H}_{22} \mathrm{O}_{4}$ & 278.34 & 7.00 \\
\hline
\end{tabular}


Table 8 . Biologically active chemical compounds of acetone extract of the aerial parts of $A$. javanica

\begin{tabular}{|c|c|c|c|c|c|c|}
\hline No & CAS & $\mathrm{RT}(\min )$ & $\begin{array}{l}\text { Name of the } \\
\text { compound }\end{array}$ & $\begin{array}{l}\text { Molecular } \\
\text { formula }\end{array}$ & $\begin{array}{c}\text { Molecular } \\
\text { weight }\end{array}$ & $\begin{array}{c}\text { Peak area } \\
(\%)\end{array}$ \\
\hline 1. & $460-19-5$ & 5.09 & Ethanedinitrile & $\mathrm{C}_{2} \mathrm{~N}_{2}$ & 52.036 & 0.29 \\
\hline 2. & $107-13-1$ & 5.243 & 2-Propenenitrile & $\mathrm{CH}_{2}=\mathrm{CHCN}$ & 53.06 & 0.04 \\
\hline 3. & $75-64-9$ & 9.3547 & 2-Propanamine, 2-methyl- & $\mathrm{C}_{4} \mathrm{H}_{11} \mathrm{~N}$ & 73.14 & 2.70 \\
\hline 4. & $75-55-8$ & 9.753 & Aziridine, 2-methyl- - incomplete name & $\mathrm{C} 3 \mathrm{H} 7 \mathrm{~N}$ & 57.09 & 6.70 \\
\hline 5. & $208-96-8$ & 12.6993 & Acenaphthylene & $\mathrm{C}_{12} \mathrm{H}_{8}$ & 152.19 & 2.10 \\
\hline 6. & $1752-30-3$ & 12.9541 & $\begin{array}{l}\text { Hydrazinecarbothioamide, 2-(1- } \\
\text { methylethylidene)- }\end{array}$ & $\mathrm{C}_{4} \mathrm{H}_{9} \mathrm{~N}_{3} \mathrm{~S}$ & 131.19 & 0.31 \\
\hline 7. & $95-06-7$ & 13.6721 & CDEC & $\mathrm{C}_{8} \mathrm{H}_{14} \mathrm{ClNS}_{2}$ & 223.78 & 10 \\
\hline 8. & $646-06-0$ & 14.9154 & 1,3-Dioxolane & $\mathrm{C}_{3} \mathrm{H}_{6} \mathrm{O}_{2}$ & 74.08 & 0.90 \\
\hline 9. & $883-20-5$ & 15.566 & Phenanthrene, 9-methyl- & $\mathrm{C}_{15} \mathrm{H}_{12}$ & 192.26 & 20.00 \\
\hline 10. & $140-29-4$ & 16.8838 & Benzyl nitrile & $\mathrm{C}_{6} \mathrm{H}_{5} \mathrm{CH}_{2} \mathrm{CN}$ & 117.15 & 11.00 \\
\hline 11. & $50-18-0$ & 17.4712 & $\begin{array}{l}\text { 2-[Bis(2-chloroethylamino)]- } \\
\text { tetrahydro-2H-1,3,2- } \\
\text { oxazaphosphorine-2-oxide }\end{array}$ & $\mathrm{C}_{7} \mathrm{H}_{15} \mathrm{Cl}_{2} \mathrm{~N}_{2} \mathrm{O}_{2} \mathrm{P}$ & 261.08 & 0.36 \\
\hline 12. & $88-89-1$ & 21.1417 & Phenol, 2,4,6-trinitro- & $\mathrm{C}_{6} \mathrm{H}_{3} \mathrm{~N}_{3} \mathrm{O}_{7}$ & 229.1 & 19.00 \\
\hline 13. & $128-37-0$ & 30.9725 & Butylated Hydroxytoluene & $\begin{array}{c}{\left[\left(\mathrm{CH}_{3}\right)_{3} \mathrm{C}\right]_{2} \mathrm{C}_{6} \mathrm{H}_{2}(\mathrm{C}} \\
\left.\mathrm{H}_{3}\right) \mathrm{OH}\end{array}$ & 220.35 & 27.00 \\
\hline 14. & $112-53-8$ & 32.7943 & 1-Dodecanol & $\mathrm{CH}_{3}\left(\mathrm{CH}_{2}\right)_{11} \mathrm{OH}$ & 186.33 & 47.00 \\
\hline 15. & $112-63-0$ & 36.6648 & $\begin{array}{c}\text { 9,12-Octadecadienoic acid }(\mathrm{Z}, \mathrm{Z}) \text {-, } \\
\text { methyl ester }\end{array}$ & $\mathrm{C}_{19} \mathrm{H}_{34} \mathrm{O}_{2}$ & 294.47 & 0.65 \\
\hline 16. & $50-06-6$ & 39.6006 & Phenobarbital & $\mathrm{C}_{12} \mathrm{H}_{12} \mathrm{~N}_{2} \mathrm{O}_{3}$ & 232.24 & 0.59 \\
\hline 17. & $103-23-1$ & 39.6591 & $\begin{array}{l}\text { Hexanedioic acid, bis(2-ethylhexyl) } \\
\text { ester }\end{array}$ & $\mathrm{C}_{22} \mathrm{H}_{42} \mathrm{O}_{4}$ & 370.56 & 3.20 \\
\hline
\end{tabular}

drug intermediates (Lin et al., 2010; de Sa et al., 2009; Meth-Cohn, 2000). Several tryptophan and tryptamine derivatives of prenylated indole compounds, and their scavenging activity for reactive oxygen species (ROS) and reactive nitrogen species (RNS) showing good activity (Estevão et al., 2010). In present study showing that methanolic extract and chloroform extract showed indole compounds and its derivative.

\section{Conclusions}

In conclusion, the methods revealed several new constituents, while one of the $A$. javanica extracts displayed comparative antioxidant activity to that of the bioactive molecule of Indole, 1H-Indole, 2-methyl-, 2,3,7,8Tetrachlorodibenzo-p-dioxin Disulfide, diphenyl. Finally, statistical analysis demonstrated certain correlations among the variables selected. The studies on phytochemical constituents of $A$. javanica are scanty. Identification of these compounds in the plant serves as the basis in determining the possible health benefits of the plant leading to further biologic and pharmacologic studies.

\section{Acknowledgements}

The authors gratefully acknowledge the financial support afforded by the United Arab Emirates University (UAEU), United Arab Emirates through Startup Grant of Dr. Abdul J. Cheruth (as PI) (Startup Grant no. \#31F040, UAEU). Authors thank the Environment Agency, Abu Dhabi for scientific collaboration and assistance provided for collection and authentication of plant samples.
Startup Grant No. \#31F040, from United Arab Emirates University as Startup Grant of Dr. Abdul J. Cheruth (as PI).

\section{References}

Al Ameri SA, Al Shaibani FY, Cheruth AJ, Al-Awad AI, Al-Yafei MA, Karthishwaran K, Kurup SS (2014). Comparative phytochemical analysis of Moringa oleifera and Moringa peregrina. Pharmacologyonline 3:216-21.

Al-Fatimi M, Wurster M, Schroder M, Lindequist M (2007). Antioxidant, antimicrobial and cytotoxic activities of selected medicinal plants from Yemen.Journal ofEthnopharmacology 111:657-666.

Atanasov AG, Waltenberger B, Pferschy-Wenzig EM, Linder T, Wawrosch C, Uhrin P, Wang L, Schwaiger S, Heiss EH, Rollinger JM (2015). Discovery and resupply of pharmacologically active plantderived natural products: a review. Biotechnology Advances 3:15821614.

Baltina LA, Flekhter OB, Nigmatullina LR, Boreko EI, Pavlova NI, NikolaevaSN, Tolstikov GA (2003).Lupane triterpenes and derivatives with antiviral activity. Bioorganic \& Medicinal Chemistry Letters 13:3549-3552.

Bors W, Heller W, Michel C, Saran M (1990). Flavonoids as antioxidants: determination of radical-scavenging efficiencies. Methods in Enzymology 186:343-355.

Brand-Williams W, Cuvelier ME, Berset CLWT (1995). Use of a free radical method to evaluate antioxidant activity. LWT- Food Science Technology 28:25-30. 
524

de Oliveira LF, Pereira BA, Gilbert B, Corrêa AL, Rocha L, Alves CR (2017). Natural products and phytotherapy: an innovative perspective in leishmaniasis treatment. Phytochemistry Reviews 16:219-233.

de Sa A, Fernando R, Barreiro EJ, Fraga M, Alberto C (2009). From nature to drug discovery: the indole scaffold as a 'privileged structure'.Mini Reviews in Medicinal Chemistry 9:782-793.

Deshmukh T, Yadav BV, Badole SL, Bodhankar SL, Dhaneshwar SR (2008). Antihyperglycaemic activity of alcoholic extract of Aerva lanata (L.) A.L. Juss. ex J.A. Schultes leaves in alloxan induced diabetic mice. Journal of Applied Biomedicine 6:81-87.

Duraipandiyan V, Ayyanar M, Ignacimuthu S (2006). Antimicrobial activity of some ethnomedicinal plants used by Paliyar tribe from Tamil Nadu, India. BMC Complementary and Alternative Medicine 6:3541.

El-Hadi MA, Barki YMN, YousifGM, Hassan SK (2010). Antiplasmodial activity of some medicinal plants used in Sudanese folk-medicine. Environmental Health Insights 4:1-6.

Emam SS (1999). Phytochemical studies on the herb Aerva javanica growing in Egypt. Bulletin-Faculty of Agriculture University of Cairo 50:488-514.

Estevão MS, Carvalho LC, Ribeiro D, Couto D, Freitas M, Gomes A, Ferreira LM, Fernandes E, Marques MM (2010). Antioxidant activity of unexplored indole derivatives: Synthesis and screening. European Journal of Medicinal Chemistry 45:4869-4878.

Halliwell B (1982). Superoxide-dependent formation of hydroxyl radicals in the presence of iron salts is a feasible source of hydroxy radicals in vivo. Biochemical Journal 205(2):461-463.

Harborne AJ (1998). Phytochemical methods aguide to modern techniques of plant analysis. Springer Science \& Business Media.

Jin Q, Jiao C, Sun S, Song C, Cai Y, Lin Y,Zhu Y (2016). Metabolic analysis of medicinal Dendrobium officinale and Dendrobium huoshanense during different growth years. PloS One 11(1):e0146607.

Judd WS, Campbell CS, KellogE, Stevens PF, Donoghue MJ (2008). Plant systematics: A phylogenetic approach, third edition. Sinauer Associates, Inc. Sunderland, MA.

Jumtee K, Bamba T, Fukusaki E (2009). Fast GC-FID based metabolic fingerprinting of Japanese green tea leaf for its quality ranking prediction. Journal of Separation Science 32:2296-2304.

Keskes H, Belhadj S, Jlail L, El Feki A, Damak M, Sayadi S, Allouche N (2017).LC-MS-MS and GC-MS analyses of biologically active extracts and fractions from Tunisian Juniperus phoenice leaves. Pharmaceutical Biology 55:88-95.

Kumar G, Karthik L, Rao KVB (2013). Phytochemical composition and in vitro antioxidant activity of aqueous extract of Aerva lanata (L.) Juss. ex Schult. Stem (Amaranthaceae). Asian Pacific Journal of Tropical Medicine 6:180-187.

Lidon F, Silva MM (2016). An overview on applications and side effects of antioxidant food additives.Emirates Journal of Food Agriculture 28:823-832.
Lin H, Tian Y, Zhang ZJ, Wu LL, Chen Y (2010). Quantification of piperazine phosphate in human plasma by high-performance liquid chromatography-electrospray ionization tandem mass spectrometry employing precolumn derivatization with dansyl chloride. Analytica Chimica Acta 664:40-48.

Lisec J, Schauer N, Kopka J, Willmitzer L, Fernie AR (2006). Gas chromatography mass spectrometry-based metabolite profiling in plants. Nature Protocols 1:387-396.

Malongane F, McGaw LJ, Mudau FN (2017). The synergistic potential of various teas, herbs and therapeutic drugs in health improvement: a review. Journal of the Science of Food and Agriculture 97(14):46794689.

Mathekaga AD, Meyer JJM (1998). Antibacterial activity of South African Helichrysum species. South African Journal Botany 64:293-295.

Meth-Cohn O, Yu CY, Lestage P, Lebrun MC, Cagniard DH, Renard P (2000). Pyridine and piperidine derivatives for treating neurodegenerative diseases. European Patent, EP 1050531 A1.

Nishimiki M, Rao NA, Yagi K (1972). The occurrence of superoxide anion in the reaction of reduced phenazine methosulfate and molecular oxygen. Biochemical and Biophysical Research Communications 46:849-853.

Robert I, Lin S (1994). Phytochemicals and antioxidants. In: Functional Foods. Springer US pp 393-449.

Samejo MQ, Memon S, Bhanger MI, Khan KM (2012). Chemical compositions of the essential oil of Aerva javanica leaves and stems. Pakistan Journal of Analytical \& Environmental Chemistry 13:48-51.

Singh R, Jain SC, Jain R (2010). Antioxidant activity of some medicinally important arid zone plants. Asian Journal of Experimental Science 23:215-221.

Srinivas P, Reddy SR (2012). Screening for antibacterial principle and activity of Aerva javanica (Burm. f) Juss. ex Schult. Asian Pacific Journal of Tropical Biomedicine 2:S838-S845.

Teklehaymanot T, Giday M(2010). Quantitative ethnobotany of medicinal plants used by Kara and Kwego semi-pastoralist people in lower Omo River Valley, Debub Omo Zone, Southern Nations, Nationalities and Peoples Regional State, Ethiopia. Journal of Ethnopharmacology 130:76-84.

Teschke R,ZhangL (2015). Chinese herbs and their molecules: Clinical and pathophysiological implications for the liver.Journal of Molecular Pathophysiology 4:85-92.

Wolfenden BS, Willson RL (1982). Radical-cations as reference chromogens in kinetic studies of one- electron transfer reactions; pulse radiolysis studies of 2,2'-azinobis-(3-ethylbenzothiazoline-6-sulfonate). Journal of the Chemical Society, Perkin Transactions 2:805-812.

Yamunadevi M, Wesely EG, Johnson M (2011). Phytochemical studies on the terpenoids of medicinally important plant Aerva lanata L. using HPTLC. Asian Pacific Journal of Tropical Biomedicine 1:S220-S225. 